\title{
An Adaptive Particle Filter Based Method for Real Time Face Tracking
}

\author{
Wei-Ming Chen, Yi-Lung Lin, Ya-Hsiung Hsieh \\ Institute of Computer Science and Information Engineering, National Ilan University, Chinese Taipei. \\ Email: wmchen@niu.edu.tw,r9943013@niu.edu.tw,r0143002@niu.edu.tw
}

Received 2013

\begin{abstract}
The video surveillance systems of recent years, usually major focus on the Human-Face of observation and detection. Human-Face is the most characteristic and prominent feature of a human, therefore, detection and tracking of Human-Face has become an important indicator of the study. This paper discusses video surveillance of public places and majors in automated face detection and face tracking. The main detection method is the use of Haar-Like Feature-based and through the Cascade classifier of the Adaboost face detection. In the tracking mechanism is based on particle filter and we modified SURF (Speeded up Robust Features) particle filter tracking, and thus enhance the detection and tracking accuracy.
\end{abstract}

Keywords: Particle Filter; Adaboost; Haar-Like Feature; SURF

\section{Introduction}

As the information technology made progress and the hardware has been improved in recent years, the calculation speed is much higher and the application is more diverse. In the image-processing field, it is easier to get images with better quality thru current hardware device and we can easily do analysis and process via images. Plus that there are a verity of multi-media products, such as digital camera, digital video, 3D digital TV, digital monitoring system, HMI, image-processing software, etc..., which makes image-processing technology more popular and important. The application of human-face detection/recognition and human-face tracking in multi-media field is getting more diverse. For example, the auto-focus of human face inside digital camera can let people take pictures with better quality. The function of human-face recognition in monitoring system or entrance security indicates the importance of human face tracking and recognition in image-processing technology.

The technology of human face recognition is to judge whether there exists human-faces or not inside a picture or video. If there exists a human face, it will locate the size and position of human face by the algorithm of human-face location. Meanwhile, it will predict the possible position of next human-face by tracking technology and associated information.

\section{Skin Color Model Face Detection}

The technology of image tracking has wide applications in many fields, such as monitoring system, robot navigation, missile tracking etc... You can find image tracking in these fields. The most common application upon image tracking is Kalman Filter. Recent years, there exists a tracking technology which can perform more efficiently \& accurately and also can deal with more complicated environment \& nonlinear Gaussian Model. This technology is called Particle Filter, which is a latest tracking method.

The direction of this thesis is the technology of human-face detection \& tracking. The technology of human-face detection is to judge whether there exists any human-face in a picture or video sequence and to locate the size \& position of human-face. The general humanface detection technology is to do detection \& tracking based on the model of skin colour, but this method will be affected by illuminations easily. This thesis uses the method of Adaboost [1] to do human face detection. In human-face tracking, we improve Particle Filter to do human-face tracking, in order to achieve a human-face tracking system with better efficiency and accuracy.

\section{Particle Filter}

We usually use Kalman Filter to track targets under complicated environment, but the target must follow Gaussian distribution by using Kalman Filter. However, there are many reasons to cause the existence of nonlinear and nonGaussian distribution in reality, such as jumping change of tracking target. This will lead to a bad tracking result. 
Particle Filter can solve the algorithm of nonlinear and non-Gaussian problems. The distribution of post probability is a group of discrete samples with weighing factor. During the process of target tracking, it will do the sampling for each frame of imaging sequence. Then, do the prediction of particle, then weigh and output, so as to predict the position of tracking target.

There are three steps of Particle Filter, i.e. sampling, deliver prediction, and measurement.

\subsection{Sampling}

In the sampling process, the particle groups with high weighing factor could be our tracking target. The higher the weighing factor is, the more similar to our tracking target the particle is. Then, we will delete the particles with smaller particles.

\subsection{Prediction}

After finishing the sample process, the particle groups with smaller weighing factor are deleted. In order to make sure the uniformity of each particle number, the particle groups with higher weighing factor will be kept. We will also make extensions on kept particles and transfer in different directions \& speeds to make predictions, in order to make sure the uniformity of possibility on each particle.

\subsection{Measurement}

The last step is to finish the prediction. We use dynamic models to predict the positions of tracking target. The new weighing factor of particle group will be obtained by applying a measuring model with high accuracy.

\section{System Architecture}

Here are the system structures of human-face detection \& tracking methods applied in this thesis. The first step is to get the image sequences of video frames. The second is to use the received image sequences to do some pre-process, in benefit to the post calculation of human-face detection and tracking. The third step is to do human-face detection by detector, then find the related information of human-face position. (Figure 1)

\subsection{The Model-Measurement Improvement of Particle Filter}

Particle filter usually uses skin color model to do human-face tracking. If human-face is shaded or the illumination changes severely, the increased tracking deviation will cause the tracking failure. So, we propose an improvement of particle filter tracking model, in order to locate the position of human face and calculate the best window of human face in position, size and angle.

\section{Process of model-measurement}

First, use skin color model to estimate human-face position, then judge whether there exists any human face in video frame. Meanwhile, we will collect the samples of particle filter. If the collected sample of human-face is lower than $30 \%$, it means to use skin-color as humanface tracking fails. Then, it will activate human-face detection mechanism to locate human-face. If the collected sample of human-face is higher than $50 \%$, it means to use skin-color as human-face tracking works well. If the collected sample of human-face is between $30 \%$ and $50 \%$, SURF mechanism will be activated to update the position of human-face. (Figure 2)

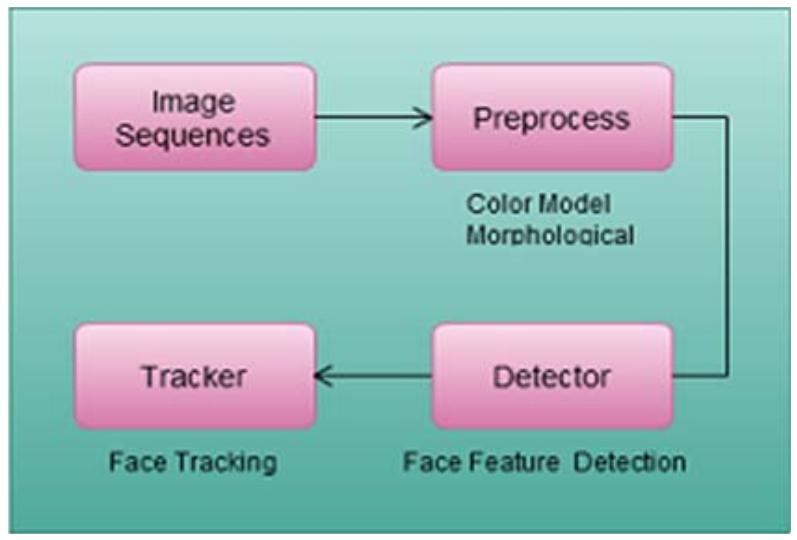

Figure 1. System architecture.

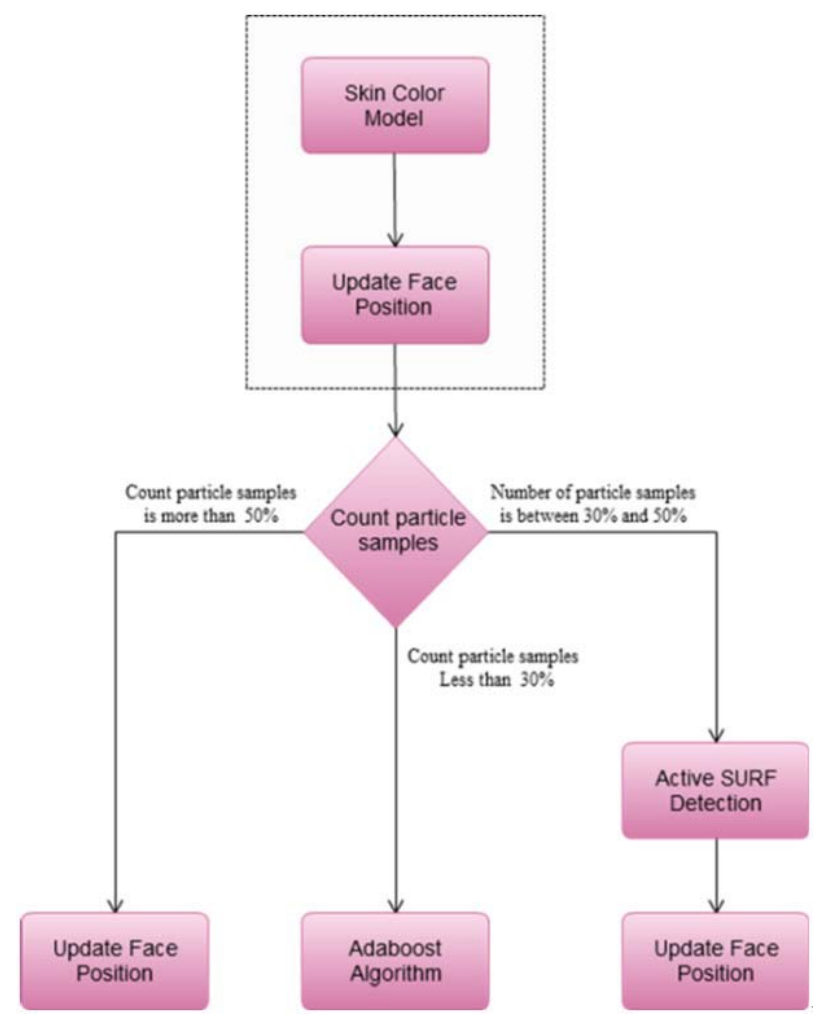

Figure 2. Process of model-measurement. 
In the step of Speeded up Robust Feature modified particle model, we will duplicate the human-face detected by Adaboost, and then receive the information of width \& height of Face ROI. In the step of human-face trakcing, we can keep using the information of Face ROI continuously until the human-face vanishes, if the tracking target of human-face does not change. In order to speed up the real-time calculation speed of SURF, we use the received width and height information of Face ROI to locate the center of human-face target, expand twice the size as Face ROI searching area, in order to lower the calculation of SURF and rise the detection rate.

\subsection{System Flowchart}

First, use vision device to get the image sequence. In order to increase the detection speed in human-face tracking, we use edge-detection rate to get rid of some Feature Set not belongs to Adaboost. By using human-face detection to locate human-face, we will copy the ROI, and then go to tracking step. While particle filter makes sampling, it will remove some confirmed non-tracking targets. Then, re-predict the possible area of human-face. In the measuring model, we improve original Skin Color Model by adding SURF to do careful human-face prediction upon measuring model. (Figure 3)

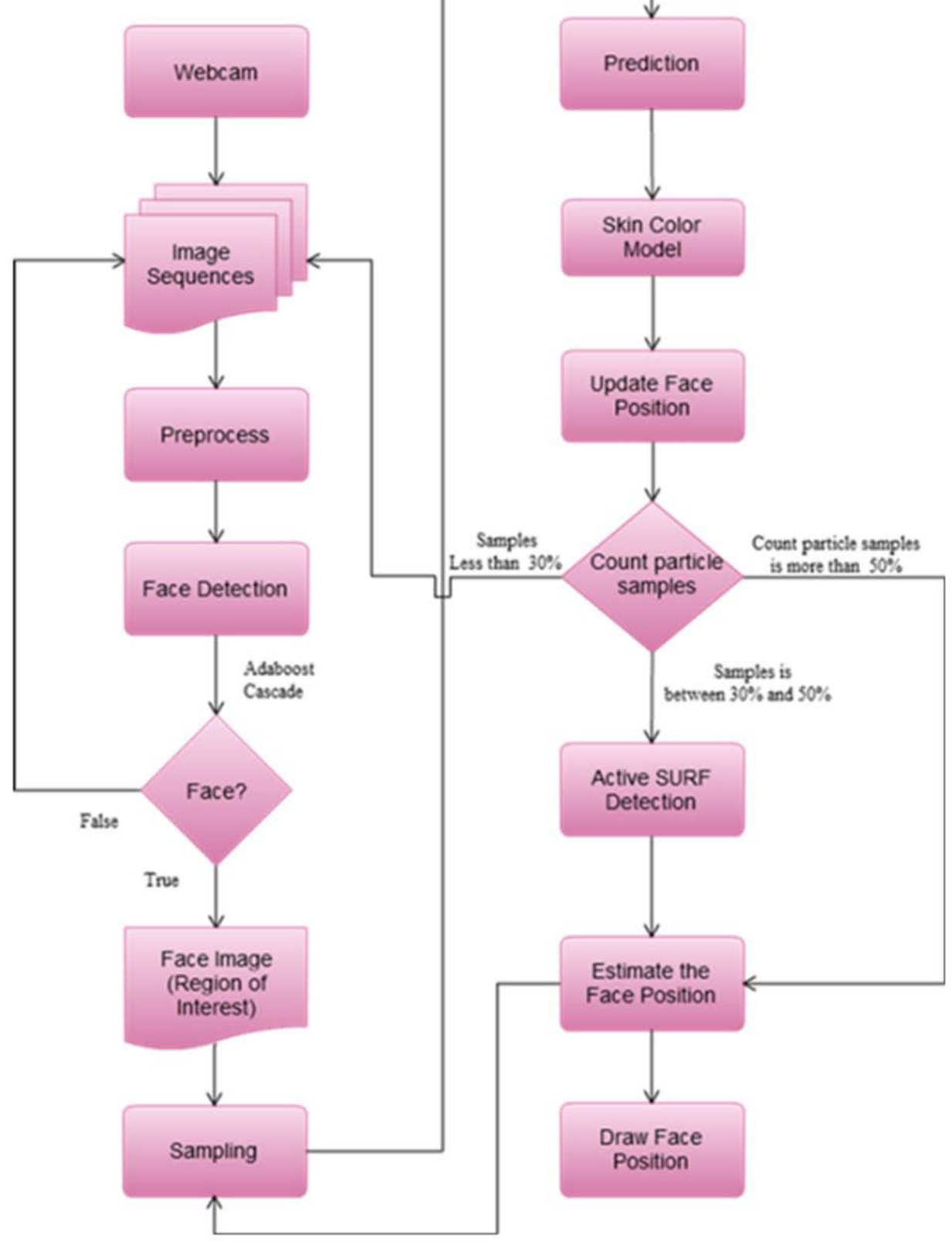

Figure 3. System flowchart. 


\section{Experimental Result}

By using image sequence of experimental test, this chapter compares Skin Color Model, here called Original Method, and our improved particle filter in efficiency. We use two image sequences to compare the results of human-face detection \& tracking.

\subsection{Translational Motion}

Image sequence of translational motion : under a complicated environment to monitor human-face to go from left to right then out of monitor area, we use this sequence to test Skin Color Model and improved particle filter added with SURF, then to compare the strength of these two methods in tracking algorithm. This video has 226 frames in about 7 seconds, moving from left to right until out-of monitor area.

By the experimental statistics of translation motion image sequence to compare Skin Color Model and improved particle filter, there exists a big vibration in Original human-face detection. It means that it does not work well in human-face tracking. By using our improved particle filter to track human-face, the tracking result is good and it can cope with human-face planar motion \& detection of human-face out-of monitor area. (Table 1)

Using Skin Color Model to do average human-face detection, the average detection rate is $59.97 \%$; using our improved particle filter method to track human face, the average detection rate of human-face is $82.31 \%$. (Figure 4 and Table 2)

\section{Discussed and Future Work}

This thesis provides a detection \& tracking system of human-face. It uses Adaboost to do human-face tracking upon video frames, and then use SURF to extract \& match features, to help particle filter do tracking and increase the accuracy of matching results in particle filter. Traditional tracking system can only track object in constant movement. While tracking object is shaded or suddenly stops or change moving direction, it will increase the difficulty of tracking. By combining particle filter and SURF, it will improve the object tracking under nonconstant motion, non-linear, and non-Gaussian environment.

By using our proposed method, it can do the humanface tracking more accurately and efficiently than traditional human-face tracking. By improving particle filter.

In the future, we will expand the applications of our human-face detection \& tracking system. We will combine the matching of facial features to do fast tracking of human-face position in image sequence, then calculate the distance between features, inclined angles, and other

\section{Table 1. Detection rate of human-face.}

\begin{tabular}{|c|c|c|}
\hline Frame & Original & Our \\
\hline 1 & $71.58 \%$ & $97.60 \%$ \\
\hline 6 & $33.26 \%$ & $90.49 \%$ \\
\hline 9 & $84.78 \%$ & $96.05 \%$ \\
\hline 12 & $65.41 \%$ & $87.00 \%$ \\
\hline 15 & $2.67 \%$ & $90.51 \%$ \\
\hline 20 & $83.25 \%$ & $90.46 \%$ \\
\hline 24 & $73.66 \%$ & $88.75 \%$ \\
\hline 28 & $57.16 \%$ & $93.07 \%$ \\
\hline 32 & $43.47 \%$ & $91.74 \%$ \\
\hline 38 & $37.00 \%$ & $84.09 \%$ \\
\hline 44 & $76.34 \%$ & $74.47 \%$ \\
\hline 137 & $35.95 \%$ & $77.05 \%$ \\
\hline 141 & $68.47 \%$ & $76.29 \%$ \\
\hline 144 & $51.04 \%$ & $70.54 \%$ \\
\hline 154 & $69.92 \%$ & $88.87 \%$ \\
\hline 157 & $37.60 \%$ & $79.75 \%$ \\
\hline 160 & $64.80 \%$ & $77.90 \%$ \\
\hline 163 & $35.06 \%$ & $76.57 \%$ \\
\hline 167 & $81.82 \%$ & $79.78 \%$ \\
\hline 170 & $72.15 \%$ & $78.70 \%$ \\
\hline 174 & $80.94 \%$ & $77.17 \%$ \\
\hline 180 & $97.46 \%$ & $74.77 \%$ \\
\hline 187 & $56.56 \%$ & $76.50 \%$ \\
\hline 190 & $60.06 \%$ & $83.42 \%$ \\
\hline 197 & $57.12 \%$ & $74.78 \%$ \\
\hline 205 & $50.53 \%$ & $82.14 \%$ \\
\hline 208 & $74.85 \%$ & $75.21 \%$ \\
\hline 215 & $70.11 \%$ & $79.65 \%$ \\
\hline 220 & $50.39 \%$ & $80.02 \%$ \\
\hline 226 & $55.66 \%$ & $75.98 \%$ \\
\hline
\end{tabular}

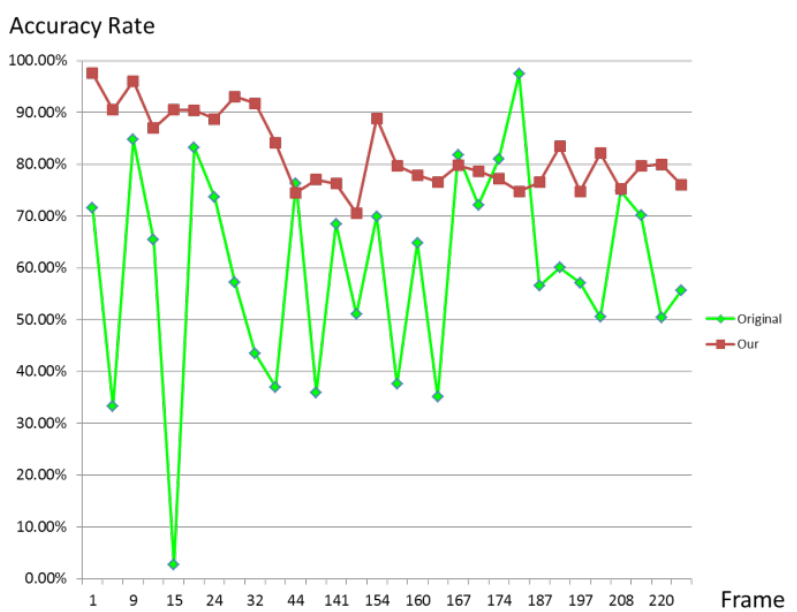

Figure 4. Translational motion detection rate of humanface. 
Table 2. Translational motion Average Accuracy Rate.

\begin{tabular}{cc}
\hline & Average Accuracy Rate \\
\hline Original & $59.97 \%$ \\
Our & $82.31 \%$ \\
\hline
\end{tabular}

related information. By extracting these information of human-face, we will increase more interactive function in later extended situation, in order to reach the interactive system of HMI.

\section{REFERENCES}

[1] R. L. Hsu, M. Abode-Mottaleb and A. K. Jain, "Face Detection in Color Images," IEEE Transactions on Pattern Analysis and Machine Intellgence, Vol. 24, No. 5, 2002.

[2] Viola and P. Jones, "Rapid Object Detection Using a Boosted Cascade of Simple Features," Proceedings of the 2001 IEEE Computer Society Conference, Vol. 1, 2001, pp. 1511- 1518.

[3] Viola and P. Jones, "Robust Real-Time Face Detection," International Journal of Computer Vision archive, Vol. 57, No. 2, 2004.

[4] Rainer Lienhart and Jochen Maydt, "An Extended Set of Haar-like Features for Rapid Object Detection,” Image Processing, Vol. 1, 2002, pp. 900-903.

doi: 10.1109/ICIP.2002.1038171

[5] Rainer Lienhart, Alexander Kuranov and Vadim Pisa- revsky, "Empirical Analysis of Detection Cascades of Boosted Classifiers for Rapid Object Detection,” MRL Technical Report, 2002.

[6] Giusto, D. D. Massidda and F. Perra, “A Fast Algorithm for Video Segmentation and Object Tracking,” Digital Signal Processing, Vol. 2, 2002, pp. 697-700.

[7] R. E. Kalman, "A New Approach to Linear Filtering and Prediction Problems,” Transactions of the ASME Journal of Basic Engineering, Vol. 82, 1960, pp. 35-45. doi: 10.1115/1.3662552

[8] G. Welch and G. Bishop, "An Introduction to the Kalman Filter," Department of Computer Science University of North Carolina at Chapel Hill, 1997, pp. 27599-3175.

[9] R. Lienhart, A. Kuranov and V. Pisarevsky, "Empirical Analysis of Detection Cascades of Boosted Classifiers for Rapid Object Detection,” MRL Technical Report, 2002.

[10] N. J. Gordon, D. J. Salmond and A. F. M. Smith, "Novel Approach to Nonlinear/non-Gaussian Bayesian State Estimation," IEEE Proceedings F Radar and Signal Processing, Vol. 12, No. 2, 1993, pp. 107-113.

[11] D. G. Lowe, “Object Recognition from Local Scale-invariant Features,” International Conference of Computer Vision, Vol. 60, 1999, pp. 1150-1157.

[12] H. Bay, T. Tuytelaars, and L. Van Gool, "SURF: Speeded up Robust Features," Proceedings of European Conference on Computer Vision, 2006, pp. 404-417.

[13] L. Juan and O. Gwun, "A Comparison of SIFT, PCA-SIFT and SURF,” International Journal of Image Processing, Vol. 3, 2009, pp. 143-152. 